\title{
Versuche uber die Vertheilung des Broms im Thierkörper nach Eingabe von Bromverbindungen. \\ Von
}

\section{Dr. Werner Rosenthal.}

(Aus dem chem. Institut der med. Fakultät zu Freiburg.)

(Der Redaction zugegangen am 1. Juni 1896.)

Angeregt durch die Entdeckung des Jodgehaltes der Schilddrüsen und durch Beobachtungen von Dr. Pflaumer') im Erlanger physiologischen Institut, nach welchen das Brom, besonders nach Gaben von Bromwasserstoffpepton, durchaus nicht so rasch ausgeschieden werde, als man bisher im Allgemeinen annahm, untersuchte ich, ob bei Hunden einverleibtes Brom in irgend einer Drüse und besonders, ob es etwa analog dem chemisch ihm so ähnlichen Jod in der Thyreoidea aufgespeichert würde. Herr Prof. Baumann gestattete mir, die Untersuchung in seinem Institute zu machen und unterstützte mich mit Rath und Hilfe, wofür ich ihm zu Dank verpflichtet bin. Ich habe nur wenige Versuche gemacht, da das Resultat aber in einer Hinsicht sicher erscheint, wird eine Mittheilung desselben wohl ron einigem Interesse sein. Aehnliche Versuche scheinen bisher nur von Nencki und Schoumow-Simanowsky') angestellt worden $\mathrm{zu}$ sein, aber auch nur in sehr geringer Zahl.

Da von vornherein anzunehmen war, dass sich in den trockenen, nur wenige Gramme wiegenden Hundeschilddrüsen

1) Erlangen, Dissertation 1895.

2) Studien über das Chlor und die Halogene im Thierkőrper. Arch. f. exper. Pathologie und Pharmakologie, Bd. 34, S. 313-333. 
nur einige Milligramme Brom finden würden, so wurde die colorimetrische Methode gewählt und zunächst so verfahren, wie Herr Prof. Baumann nach Rabourdin's Vorgang das Jod in Schilddrüsen nachweist '), nur dass zur schliesslichen Abscheidung des Broms Chlorwasser verwandt wurde. Dies Verfahren erwies sich aber als sehr wenig empfindlich für Brom, im Gegensatz zur Jodreaction, hauptsächlich wohl wegen der leichteren Löslichkeit des Broms in Wasser. Ich verfuhr desshalb bei meinen Analysen nach folgender Modifikation.

Die mit Alkali und Salpeter gewonnene Schmelze der Substanz wurde gelöst und aus der mit Salpetersäure angesäuerten Lösung die Halogene mit Silbernitrat gefällt. Der Niederschlag wurde durch ein kleines Filter abfiltrirt und ausgewaschen, nach Durchstossen des Filters in ein kleines Bechergläschen gespült und das Spülwasser durch Decantiren oder Eindampfen auf 5-10 cbcm. eingeengt. Darauf wurde der Halogensilberniederschlag mit Zinkstaub gut vermengt und durch Zufügen von 1-3 Tropfen verdünnter Schwefelsäure Bromwasserstoff abgeschieden. Nach mehrmaligem Umrühren und längerem Stehen wurde vom Rückstand in ein kalibrirtes Röhrchen mit flachem Boden abfiltrirt, der Rückstand zunächst in dasselbe, dann in ein anderes Röhrchen mit einigen cbcm. angesäuerten Wassers ausgewaschen. Im Filtrat wurde durch tropfenweises Zufügen von Chlorwasser und Ausschütteln mit Schwefelkohlenstoff zunächst etwa vorhandenes Jod und danach das Brom abgeschieden. Das besonders aufgefangene Spülwasser diente zur Kontrolle, ob keine merklichen Mengen Brom im Rückstand zurückgehalten würden. Die Proben wurden immer mit genau $1 \mathrm{cbcm}$. Schwefelkohlenstoff ausgeschüttelt und die Menge des abgeschiedenen Broms geschätzt durch Vergleich mit Proben einer 1\% Bromkaliumlösung, welche mit Wasser zur gleichen Flüssigkeitsmenge aufgefüllt, mit einigen Tropfen Schwefelsäure angesäuert und tropfenweise mit Chlorwasser versetzt wurden.

1) Diese Zeitschrift, Bd, XXI, S. 489. 
Einige Kontrollversuche ergaben, dass sich 0,2 mgr. Bromkalium in etwa $10 \mathrm{cbcm}$. Wasser gelöst, auf diese Weise gerade noch durch leise Bräunung gereinigten Schwefelkohlenstoffs erkennen lassen. Bei Werthen um $1 \mathrm{mgr} . \mathrm{Br} \mathrm{K}$ in der gleichen Flüssigkeitsmenge kann man bei einiger Uebung die Unterschiede der Färbung für $1 / 10 \mathrm{mgr}$. mehr oder weniger noch gut erkennen. Bei grösseren Brommengen und dunklerer Färbung des Schwefelkohlenstoffs wird die Schätzung aber sehr unsicher.

Einige andere Versuche wurden so ausgeführt, dass je 1 gr. getrocknetes Fibrin oder Fleisch mit der $1 / 1000$ Br K-Lösung angefeuchtet und nun wie oben geschildert behandelt wurde. Es zeigte sich, dass sich bei Zufügen von nur $1 / 2 \mathrm{mgr}$. $\mathrm{BrK}$ letzteres zu $80 \%$ nachweisen liess, die Verluste aber bei grösserem Bromgehalt (von 1-5 mgr.) auf $40 \%$ stiegen. Die Methode erscheint daher zum Bromnachweis wohl brauchbar, zur Bromschätzung aber recht mangelhaft, doch immerhin genügend zur Untersuchung, ob in verschiedenen Organen und Geweben sehr verschiedene Mengen Brom zurückgehalten würden.

Ich stellte an 4 Hunden Versuche an. Da es nach den Pflaumer'schen Angaben möglich schien, dass sich das Brom im Thierkörper wesentlich verschieden verhalte, je nachdem es als Bromkalium oder als $\mathrm{P}$ a al'sches Bromwasserstoffpepton zugeführt würde, verfütterte ich an je 2 Hunde äquivalente Brommengen in Form der beiden Präparate ${ }^{1}$ ) und zwar einmal 1,6 gr. $\mathrm{Br}$ in einmaliger Dosis und das andere Mal 3,61 gr. $\mathrm{Br}$ in 6 Tagesdosen. Das 1. Paar Hunde wurde am 6. Tage nach der Bromgabe, das 2. Paar am 3. Tag nach der letzten Dosis getödtet. Das Futter bestand bei allen 4 Thieren aus Hackfleisch, in welchem die Bromdosen verfüttert wurden, daneben aus Hundekuchen und gelegentlich etwas Milch. Eine toxische Wirkung der Bromgaben wurde bei keinem der Thiere beobachtet.

Von den mit Cyankalium vergifteten Thieren wurden die Organe entnommen, möglichst blutfrei frisch gewogen, zer-

1) Das Bromwasserstoffpepton von $24 \%$ Bromgehalt wurde mir von Herrn Prof. Paal in Erlangen freundlichst zur Verfügung gestellt.

Zeitschrift für physiologische Chemie. XxII. 
kleinert, im Trockenschrank getrocknet und in je 1 gr. der Trockensubstanz die Brommenge wie oben angegeben geschätzt. Wo sich grössere Brommengen fanden, wurde die Analyse mit $1 / 2$ gr. wiederholt, sonst, soweit noch Material vorhanden war, wieder mit $1 \mathrm{gr}$. Substanz.

Bei der Unsicherheit der Methode und den vielerlei Einflüssen, welche Gewichtsunterschiede der Hunde, verschieden starke Diurese bei denselben und ähnliche Bedingungen auf das Resultat haben müssen, glaube ich die Ergebnisse nur zum geringsten Theil zahlenmässig geben zu sollen. Zunächst fand ich keinen bedeutenden Unterschied zwischen Bromwasserstoffpepton und Bromkalium; doch sei erwähnt, dass in beiden Doppelversuchen die Gesammtmenge des in der Leber zurückgehaltenen Broms bei den mit Bromwasserstoffpepton gefütterten Hunden grösser war, und zwar einmal um $1 / 3$, das an-. dere Mal um $\%$ der in der Bromkaliumleber gefundenen Menge.

In den Schilddrüsen wurde bei allen 4 Hunden Brom nachgewiesen. In 3 normalen Schilddrüsen fand es sich neben Jod in geringer Menge. Die Analysen sind hier besonders unsicher, weil die wenige verfügbare Substanz keine Kontrollversuche zuliess, doch scheint der procentuale Bromgehalt dem bei denselben Hunden in der Milz gefundenen zu entsprechen: Bei dem vierten Hunde war ein parenchymatöser Kropf vorhanden. Die beiden Thyreoidallappen des 91/, kgr. schweren Hundes wogen frisch $26 \%$ gr., trocken 5,65 gr. Jod liess sich darin nicht nachweisen, obgleich der Hund in den letzten 6 Tagen vor der Tödtung wie die anderen jodhaltigen Hundekuchen ${ }^{4}$ ) erhalten hatte. Brom fand ich aber in nicht unbedeutender Menge in seinem Kropf, nämlich mindestens 8,22 mgr. in der ganzen Schilddrüse, procentual jedoch auch hier etwa ebensoviel wie in der Milz desselben Hundes, nämlich 1,4 mgr. auf $1 \mathrm{gr}$. Trockensubstanz. Eine besondere Ansammlung von Brom in der Schilddrüse findet also nicht statt.

Eine vergleichende Schätzung des Bromgehaltes in einer grösseren Zahl von. Organen habe ich nur bei den 2 Hunden

1) Vgl. E. Ba umann, diese Zeitschr., Bd. XXII, S. 15. 
mit grösserer Bromgabe durchgeführt. Hier sind die Verhältnisse auch einigermaassen vergleichbar mit den beiden von Nencki und Schoumow-Simanowsky angestellten Versuchen von Bromfütterung bei Hunden. Zufällig haben jene in dem einen ihrer Fälle die Analysen fast nur von solchen Organen veröffentlicht, die ich nicht untersuchte. So bleiben ein Fall von jenen und 2 von mir zum Vergleich. Dabei zeigt sich nun, dass in allen 3 Fällen die Nieren den höchsten Gehalt an Brom auf 1 gr. Trockensubstanz zeigten, was nicht verwunderlich ist, da die Thiere während lebhafter Bromausscheidung getödtet wurden. Bei meinen zwei Hunden kommt nun aber die Leber darin der Niere sehr nahe und die gesammte in den grossen Lebern aufgespeicherte Brommenge ist recht bedeutend, während Nencki und Schoum owSimanowsky gerade in der Leber nur sehr geringe Brommengen fanden und in der Galle gar kein Brom nachweisen konnten. Eine Discussion über die möglichen Gründe dieses Unterschiedes scheint mir bei dem geringen Material nicht angebracht. Aber es wird von Interesse sein und vielleicht zur Verfolgung dieser Frage anregen, wenn ich in einer kleinen Tabelle die von Nencki und Schoumow-Simanowsky und die von mir gefundenen Werthe nebeneinander stelle. Dabei hebe ich hervor, dass jene auf gewichtsanalytischer Methode (nach E. Berglund), die meinen aber auf colorimetrischer Schätzung beruhen und dem ganzen Verfahren nach wohl alle bedeutend $\mathrm{zu}$ klein sind, also nur benutzt werden dürfen, um Verhältnisszahlen für den Bromgehalt der einzelnen Organe zu gewinnen.

Der von Nencki und Schoumow-Simanowsky untersuchte Hund wog $23 \mathrm{kgr}$. und erhielt 34,12 gr. Brom in Form von Bromnatrium in 10 Tagesdosen; 3 Tage nach der letzten wurde er getödtet. Meine Hunde wogen 8,2 und 9,25 kgr., erhielten 3,61 gr. Br als Bromkalium und Bromwasserstoffpepton in 6 Tagesdosen und wurden 2 Tage nach der letzten getödtet. Die Bromdosen pro kgr. Körpergewicht. waren also 2,33 gr. bei dem Nencki und SchoumowSimanowsky'schen Hund, 0,44 gr. bei dem Brompepton- 
und 0,39 gr. bei dem Bromkaliumhund. Die Brommengen in Procenten der Trockensubstanz betragen bei den 3 Hunden in der:

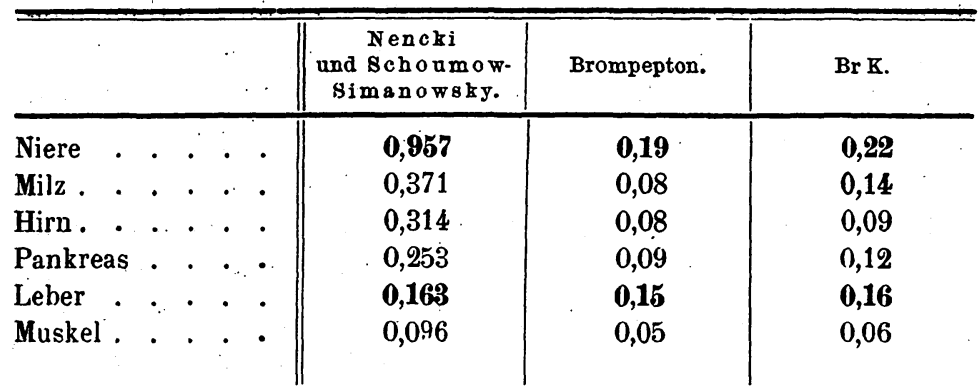

Dem aufmerksamen Leser mag auffallen, dass laut dieser Tabelle der mit Bromkalium gefütterte Hund bei kleinerer Bromgabe pro kgr. Körpergewicht mehr Brom im Gramm. Trockensubstanz der Leber enthält als der mit Bromwasserstoffpepton gefütterte, während ich oben doch anführte, dass Letzterer $1 / 8$ Brom mehr in der Leber aufgespeichert hätte als Ersterer; es erklärt sich daraus, dass der um $1 \mathrm{kgr}$. leichtere Hund eine 55 gr. schwerere Leber hatte (302 gr.: 247 gr.). Dies Beispiel zeigt wie verwickelt die bei solchen Versuchen in Betracht kommenden Verhallnisse sind.

Vermuthlich lassen sich andere und vielleicht interessantere Ergebnisse erhalten, wenn man die Organe längere Zeit nach den Bromgaben untersucht. Darauf deutet der Befund bei dem von mir mit einer einmaligen Dosis von $5,6 \mathrm{gr}$. Brompepton (=1,35 gr. Brom) gefütterten und nach 5 Tagen getödteten Hund: hier fand sich in der Leber 1,2 mgr. Brom auf 1 gr. Trockensubstanz, in der Niere nur etwas mehr als 0,5 mgr. und in der Milz fast ebensoviel. Eine vergleichende Untersuchung anderer Organe desselben Hundes oder der Organe des mit der entsprechenden Bromkaliumdosis gefütterten habe ich leider versäumt. 